\title{
Covering the proximal nerve stump with chondroitin sulfate proteoglycans prevents traumatic painful neuroma formation by blocking axon regeneration after neurotomy in Sprague Dawley rats
}

\author{
${ }^{*}$ Fu-Lin He, MPhil,1,2 Shuai Qiu, MD, PhD, ${ }^{1,2}$ Jian-Long Zou, PhD, ${ }^{3}$ Fan-Bin Gu, MPhil,1,2 \\ Zhi Yao, MD, PhD, ${ }^{1,2}$ Zhe-Hui Tu, MD, PhD, ${ }^{1}$ Yuan-Yuan Wang, MD, PhD, ${ }^{1}$ Xiao-Lin Liu, MD, PhD, 1,2,4 \\ Li-Hua Zhou, PhD, ${ }^{5}$ and Qing-Tang Zhu, MD, PhD ${ }^{1,2,4}$ \\ ${ }^{1}$ Department of Microsurgery and Orthopedic Trauma, First Affiliated Hospital of Sun Yat-sen University, Guangzhou; ${ }^{2}$ Center \\ for Peripheral Nerve Tissue Engineering and Technology Research; ${ }^{3}$ School of Basic Medical Sciences, Guangzhou Medical \\ University; ${ }^{4}$ Guangdong Province Engineering Laboratory for Soft Tissue Biofabrication; and ${ }^{5}$ Department of Anatomy, School of \\ Medicine, Sun Yat-sen University, Guangzhou, Guangdong Province, China
}

\begin{abstract}
OBJECTIVE Neuropathic pain caused by traumatic neuromas is an extremely intractable clinical problem. Disorderly scar tissue accumulation and irregular and immature axon regeneration around the injury site mainly contribute to traumatic painful neuroma formation. Therefore, successfully preventing traumatic painful neuroma formation requires the effective inhibition of irregular axon regeneration and disorderly accumulation of scar tissue. Considering that chondroitin sulfate proteoglycans (CSPGs) can act on the growth cone and effectively inhibit axon regeneration, the authors designed and manufactured a CSPG-gelatin blocker to regulate the CSPGs' spatial distribution artificially and applied it in a rat model after sciatic nerve neurectomy to evaluate its effects in preventing traumatic painful neuroma formation.
\end{abstract}

METHODS Sixty female Sprague Dawley rats were randomly divided into three groups (positive group: no covering; blank group: covering with gelatin blocker; and CSPG group: covering with the CSPG-gelatin blocker). Pain-related factors were evaluated 2 and 8 weeks postoperatively $(n=30)$. Neuroma growth, autotomy behavior, and histological features of the neuromas were assessed 8 weeks postoperatively $(n=30)$.

RESULTS Eight weeks postoperatively, typical bulb-shaped neuromas did not form in the CSPG group, and autotomy behavior was obviously better in the CSPG group $(p<0.01)$ than in the other two groups. Also, in the CSPG group the regenerated axons showed a lower density and more regular and improved myelination $(p<0.01)$. Additionally, the distribution and density of collagenous fibers and the expression of $\alpha$-smooth muscle actin were significantly lower in the CSPG group than in the positive group $(p<0.01)$. Regarding pain-related factors, $c$-fos, substance $P$, interleukin (IL)-17, and IL-1 $\beta$ levels were significantly lower in the CSPG group than those in the positive and blank groups 2 weeks postoperatively $(p<0.05)$, while substance P and IL-17 remained lower in the CSPG group 8 weeks postoperatively $(p<0.05)$.

CONCLUSIONS The authors found that CSPGs loaded in a gelatin blocker can prevent traumatic neuroma formation and effectively relieve pain symptoms after sciatic nerve neurotomy by blocking irregular axon regeneration and disorderly collagenous fiber accumulation in the proximal nerve stump. These results indicate that covering the proximal nerve stump with CSPGs may be a new and promising strategy to prevent traumatic painful neuroma formation in the clinical setting.

https://thejns.org/doi/abs/10.3171/2020.3.JNS193202

KEYWORDS chondroitin sulfate proteoglycans; neuropathic pain; traumatic neuroma; peripheral nerve injury ABBREVIATIONS CSPG = chondroitin sulfate proteoglycan; DRG = dorsal root ganglia; IL = interleukin; PNS = proximal nerve stump; RT-PCR = real-time polymerase
chain reaction; SD = Sprague Dawley; TBST = Tris-buffered saline Tween; TEM = transmission electron microscopy; $\alpha$-SMA = $\alpha-$ Smooth muscle actin.

SUBMITTED November 24, 2019. ACCEPTED March 6, 2020.

INCLUDE WHEN CITING Published online May 29, 2020; DOI: 10.3171/2020.3.JNS193202.

${ }^{*}$ F.L.H. and S.Q. contributed equally to this study. 
$\mathrm{A}$ TRAUMATIC neuroma is a tangle of irregular nerve fibers and disorganized collagen connective tissue that mainly develops from failed nerve regeneration, such as in cases of peripheral nerve injuries or after amputation surgery. ${ }^{1}$ The regenerated axons trapped in the disorganized collagenous fibers show abnormal and increased excitability, which may render them sensitive to ectopic stimuli, ultimately leading to spontaneous neuropathic pain and abnormal evoked pain., ${ }^{2,3}$ For example, among patients who undergo amputation, approximately $7.8 \%$ and $4.1 \%$ of patients who undergo initial digit amputation and lower-limb amputation, respectively, develop a symptomatic neuroma, which mainly causes abnormal hyperpathia or intractable pain, thus decreasing the quality of life of these patients. ${ }^{4-6}$ Therefore, the prevention of traumatic painful neuroma formation is important and necessary for those who suffer from peripheral nerve injuries or undergo amputation.

The exact mechanisms of traumatic painful neuroma formation in the peripheral nervous system are not yet fully understood. ${ }^{2}$ Some studies have shown that in neuromas, a high proportion of immature or unmyelinated regenerated axons and the high expression of $\alpha$-smooth muscle actin ( $\alpha$-SMA) and neurotrophins, such as nerve growth factor or brain-derived neurotrophic factor after neurotomy, play certain roles in traumatic painful neuroma formation. ${ }^{7-10}$ In addition, activation of the RhoA/ROCK signaling pathway contributes to the process of traumatic painful neuroma formation after neurectomy because this pathway can effectively suppress axon outgrowth. ${ }^{11}$ However, the neuropathic pain caused by traumatic neuroma is a complicated subject, and the exact mechanisms still require further investigation.

Currently, a variety of methods can be used to prevent the formation of traumatic neuromas, such as simple excision of the neuroma, insertion of the proximal nerve stump (PNS) into an adjacent anatomical structure, or covering the PNS with epineurium or a tissue-engineered cap, ${ }^{12}$ but there is still no uniformly successful treatment for symptomatic neuromas in the clinic, especially those in patients with chronic intractable pain, because of the regenerative capacity of the peripheral nervous system. For example, it has been reported that the reoperation rate reaches $65 \%$ when the surgeon has performed only a simple symptomatic neuroma excision, which explains the use of additional methods. ${ }^{13}$ Therefore, new methods and strategies should be exploited to thoroughly prevent the formation of traumatic painful neuromas, and it is especially essential to block irregular axon regeneration in neuromas.

Chondroitin sulfate proteoglycans (CSPGs), composed of a protein core and chondroitin sulfate side chains, are components of the extracellular matrix in the nervous system, where they play positive and necessary roles in axon guidance during nervous system development. ${ }^{14,15}$ Extensive evidence shows that CSPGs are major inhibitors of neurite outgrowth after nerve injuries through the RhoA/ ROCK signaling pathway, which is activated to promote growth cone collapse and to inhibit growth cone regrowth, by binding to the CSPG receptors distributed in growth cones, such as NgR1 and NgR3. ${ }^{16,17}$ After nerve injuries, CSPGs diffusely and randomly distribute at the injury site and in other scar tissue, such as disorganized connective fiber tissue, the main component of traumatic neuromas. This kind of disordered distribution may aggravate the disorder of regenerated axons and then cause neuropathic pain. Therefore, the spatial distribution of CSPGs should be artificially adjusted to effectively inhibit axon outgrowth. Once the distribution of CSPGs becomes regular, CSPGs may play unexpected roles in preventing the formation of traumatic painful neuromas.

Gelatin is a biodegradable material with good histocompatibility, a property for which gelatin is widely applied in biological tissue engineering, and the physical and biological properties of gelatin can be further optimized by crosslinking with genipin. Therefore, in our study, we designed and successfully manufactured a CSPG-gelatin blocker and then applied the blocker to the proximal sciatic nerve stump after neurotomy in Sprague Dawley (SD) rats. We hypothesized that the CSPG-gelatin blocker could effectively suppress the outgrowth of new axons and reduce irregular axon regeneration, inducing a "blocking signal" for axon regeneration to inhibit the process of traumatic painful neuroma formation.

\section{Methods}

\section{Preparation of the CSPG-Gelatin Blocker}

Briefly, we dissolved $800 \mathrm{mg}$ of gelatin (Bloom, 900070-8; Aladdin) and $10 \mathrm{mg}$ of genipin (98\%, 6902-778; Aladdin) in $10 \mathrm{ml}$ of ultrapure water, followed by the dissolution of $200 \mu \mathrm{g}$ of CSPGs (CC117; Millipore) in the aforementioned mixed solution. In the blank group, no CSPGs were added; instead, an equal amount of ultrapure water was added to the gelatin solution. After vibrating and fully mixing the solution, we injected the mixed solution into the blocker mold and then placed the mold in a $37^{\circ} \mathrm{C}$ incubator for 24 hours to achieve full crosslinking. Subsequently, the mold was moved to a $-80^{\circ} \mathrm{C}$ refrigerator for 10 minutes to shape, after which the blocker was unloaded from the mold. Finally, the CSPG-gelatin blocker was freeze dried for 12 hours, and cobalt radiation, at a dose of a $25 \mathrm{kGy}$, was used to sterilize the blocker before it was applied in the animal experiment. Scanning electron microscopy was used to observe the microstructure and dot blot analysis was used to evaluate CSPG activity and concentration (Fig. 1). Briefly, standard CSPG solutions ( 0 , 10,20 , and $30 \mu \mathrm{g} / \mathrm{ml}$ ) and solutions extracted from blank gelatin blockers or CSPG-gelatin blockers were prepared. Then, $2 \mu \mathrm{l}$ of each solution was spotted onto a nitrocellulose membrane (Trans-Blot; Bio-Rad) and completely dried. After blocking the nonspecific sites in the membrane by soaking in $5 \%$ bovine serum albumin in Tris-buffered saline Tween (TBST) buffer (20 mM Tris-HCl, $150 \mathrm{mM}$ $\mathrm{NaCl}, \mathrm{pH} 7.5,0.05 \%$ Tween 20) for 1 hour at room temperature, the membrane was incubated with a mouse monoclonal anti-chondroitin sulfate antibody (dilution: 1:400, C8035; Sigma-Aldrich) for 30 minutes at room temperature. Then, the membrane was washed 3 times with TBST, after which it was incubated with goat $\mathrm{F}\left(\mathrm{ab}^{\prime}\right) 2$ anti-mouse IgG+IgM+IgA (dilution: 1:1000, ab6006; Abcam) for 30 minutes at room temperature. Then, the membrane was washed an additional 3 times with TBST and incubated 

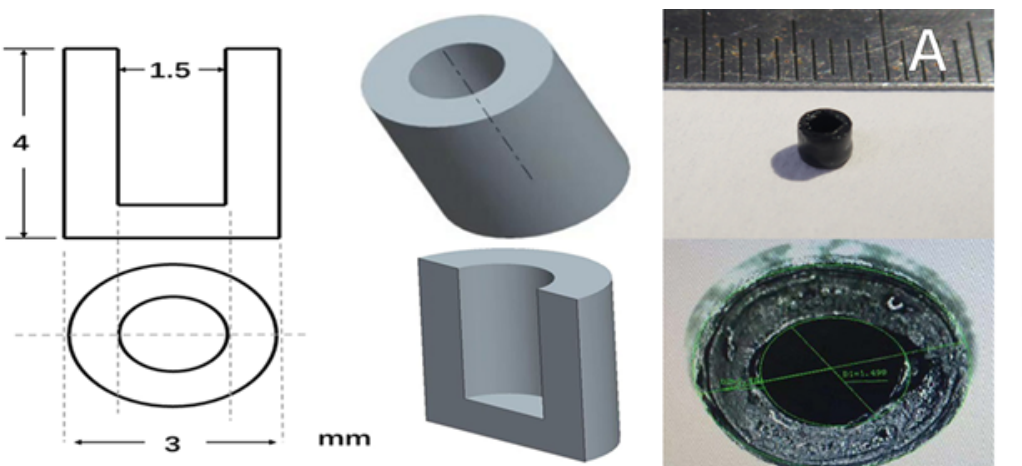

CSPGs standard solution $(\mu \mathrm{g} / \mathrm{ml}) \quad$ CSPGs-gelatin blocker
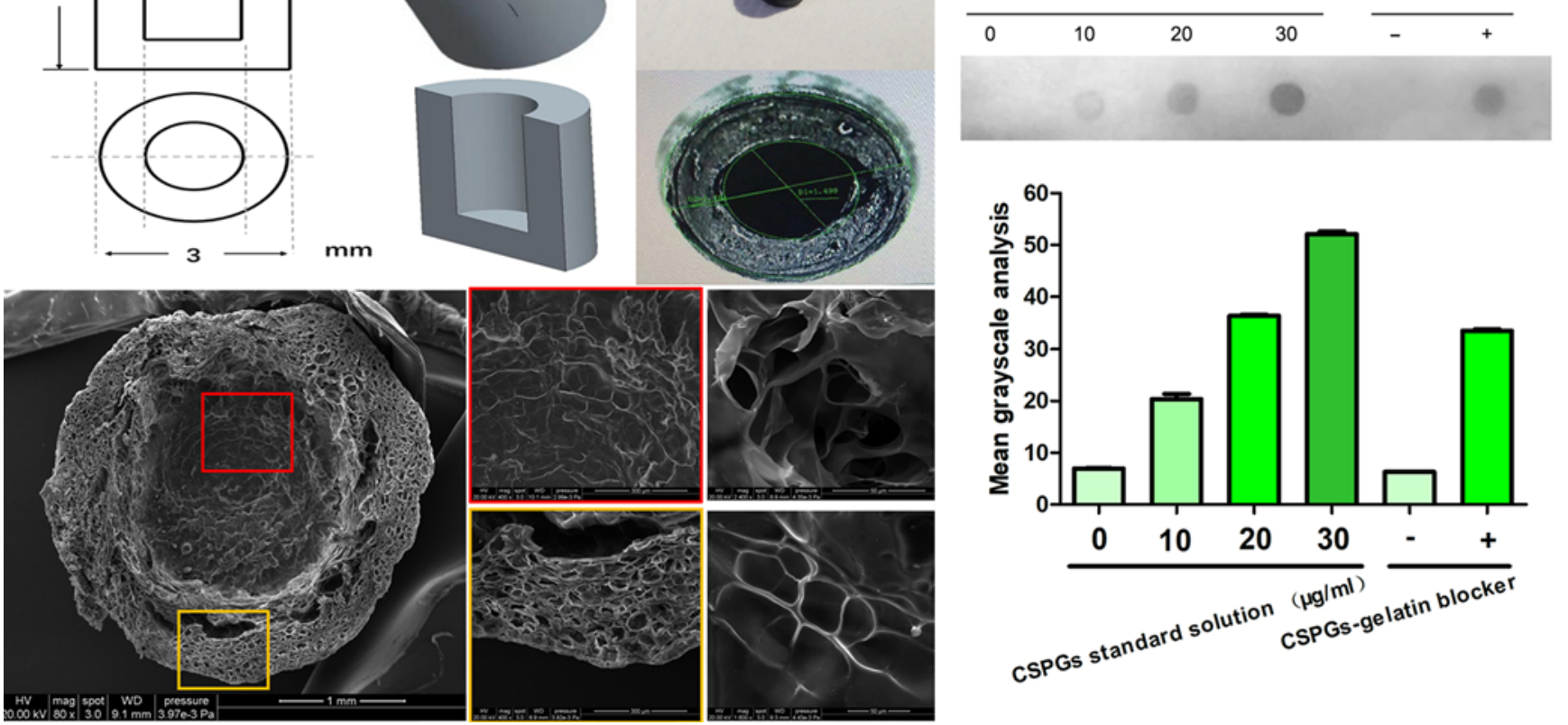

FIG. 1. Design and fabrication of the CSPG-gelatin blocker. A: Original design, actual size (D1, inner diameter, 1.499 mm; D2, outer diameter, $2.78 \mathrm{~mm}$ ), and the microstructure of the CSPG-gelatin blocker. B: Dot blot analysis for the CSPGs. From the results, we could conclude that the CSPG concentration in the gelatin was close to $20 \mathrm{mg} / \mathrm{ml}$. Figure is available in color online only.

with a Western blot chemiluminescence reagent for $1 \mathrm{~min}-$ ute. Finally, the x-ray film was exposed in the dark room.

\section{Animal Models and Grouping}

Sixty female SD rats, weighing 200-250 g and obtained from the Experimental Animal Center of the First Affiliated Hospital of Sun Yat-sen University (Guangzhou, China), were used in this study. The rats were randomly divided into three groups: positive group $(n=20)$, blank group $(n=20)$, and CSPG group $(n=20)$. All experiments were approved by the Animal Ethics Committee of the First Affiliated Hospital of Sun Yat-sen University. Animals were treated according to the National Research Council's guidelines for the care and use of laboratory animals and had free access to rat chow and water.

\section{Surgical Procedures}

Sodium pentobarbital $(50 \mathrm{mg} / \mathrm{kg})$ via an intraperitoneal injection was used to anesthetize the rats, and the right sciatic nerve was exposed under aseptic conditions. To determine the location of the neuroma and to quantitatively analyze neuroma growth, the original branch of the posterior gluteal nerve around the level of the sciatic notch was identified and labeled with an 8-0 suture under a microscope. The sciatic nerve was then sharply transected $1 \mathrm{~cm}$ from the labeled site and separated from the surrounding tissue up to the labeled site. In all cases, the distal nerve stumps (at least $1.2 \mathrm{~cm}$ ) were removed and discarded. After neurotomy, blank blockers (without CSPGs) and CSPG blockers were sutured to the PNS in the blank group and
CSPG group, respectively, with a single epineurial 10-0 monofilament nylon suture, while no management of the PNS was performed after transection in the positive group (Fig. 2). In all groups, the muscles and skin incisions were closed with 4-0 sutures.

\section{Specimen Preparation}

All rats in each group were randomly divided into two subgroups and sacrificed at 2 weeks $(n=15)$ and 8 weeks $(n=45)$ after surgery. The rats in the 2-week subgroup $(n=$ 15) were used for quantitative real-time polymerase chain reaction (RT-PCR), Western blot analysis, and ELISA. The rats in the 8-week subgroup $(n=45)$ were used for behavioral and histological analyses in addition to the items tested in the 2-week subgroup. Fifteen rats were used to run the tests performed in the 2-week subgroup, and half of the remaining 30 rats were harvested for histological studies (Masson's trichrome staining and silver staining) and immunohistochemical analysis of $\alpha$-SMA expression in the neuromas. From the other 15 rats, an approximately $1 \times 1-\mathrm{mm}$ segment was harvested from the center of the neuroma under microscopy for toluidine blue staining and transmission electron microscopy (TEM).

All surgical procedures were performed after the rats were anesthetized with an intraperitoneal injection of sodium pentobarbital $(50 \mathrm{mg} / \mathrm{kg})$; then, we collected blood samples $(1.5 \mathrm{ml})$ from the tails of all rats. The samples were placed at $4{ }^{\circ} \mathrm{C}$ overnight and centrifuged for $20 \mathrm{~min}$ utes at $2000 \mathrm{rpm}$. Then, we collected the upper sera for the ELISA study. In addition, the operative and contralat- 


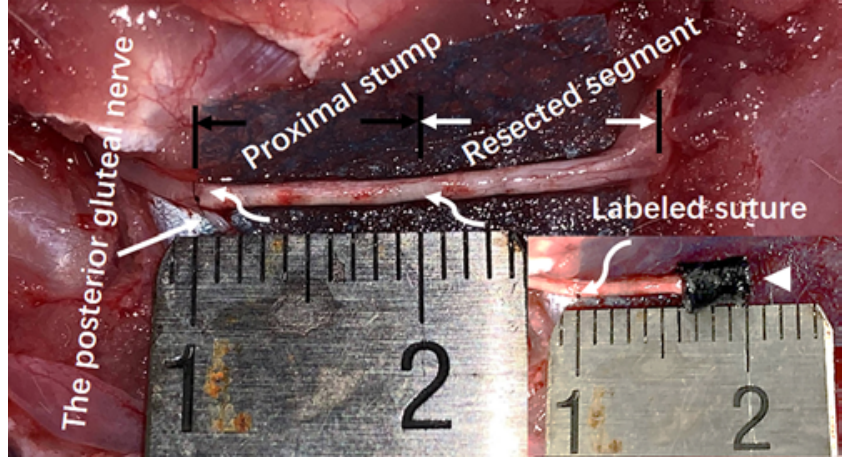

FIG. 2. Demonstration of the operation. The right sciatic nerve of each SD rat was used to establish a model of traumatic neuroma formation by neurotomy. The curved arrows on the left and at the center show the sites of the suture labels for quantitative analysis (top) and the origin of the posterior gluteal nerve (bottom, straight arrow on the left), respectively. The PNS stump was defined as $1 \mathrm{~cm}$, while the resected segment above was $1.2 \mathrm{~cm}$. The arrowhead in the inset shows the blocker sutured to the PNS after removal of the sciatic nerve. Figure is available in color online only.

eral L4-5 dorsal root ganglia (DRG) were harvested for RT-PCR analysis of RhoA gene expression, and the dorsal horn of the fourth lumbar spinal cord (located by the L4 nerve root) was harvested for Western blot analysis of cfos expression. At the same time, the neuromas were harvested to analyze the expression of substance $P$, which was used as a pain marker. All of the specimens for RT-PCR, Western blot analysis, and ELISA testing were stored at $-80^{\circ} \mathrm{C}$. The specimens used for histological analysis and TEM were preserved in $4 \%$ paraformaldehyde. All rats were euthanized by the injection of an overdose of sodium pentobarbital $(50 \mathrm{mg} / \mathrm{kg})$.

\section{Behavioral Analysis}

The rats in the 8 -week subgroup $(n=45)$ were monitored by two blinded observers to evaluate autotomy behavior (autotomy scores were recorded 2 times/week). A modified Wall scale ${ }^{18,19}$ was adopted to assign points according to the severity of autotomy. Briefly, 1 point was assigned for 2 or more toenails (maximum, 1 point per limb), and 1 point was assigned for each half digit (distal and proximal phalanges) for a possible maximum of 10 points per limb.

\section{Evaluation of Neuroma Growth}

To precisely analyze differences in growth among the groups, we labeled the sciatic nerve $1 \mathrm{~cm}$ from the transection site with an 8-0 suture, where the specimen would be harvested at the end of the experiment. The corresponding 1-cm-long segment of the sciatic nerve at the contralateral site was obtained for comparison. Then, the neuroma growth was evaluated by a weight ratio (WR) using the following formula: [weight of the neuroma (NW, after removing the blocker) - weight of the excised normal nerve segment $(\mathrm{NNW})] / \mathrm{NNW}$.

\section{Histological and Immunohistochemical Analyses}

The specimens were processed by standard paraffinembedding methods and then cut into $4-\mu \mathrm{m}$ sections. To standardize the site of staining or immunohistochemistry, sections were randomly selected between approximately 200 and $300 \mu \mathrm{m}$ from the distal end of the neuromas. At least 20 sections were obtained from each sample after excluding small sections or poorly cut sections. Then, 50 sections were randomly selected, and every fifth section was used for Masson's trichrome staining to label collagenous fibers, silver staining to label regenerative axons, or immunohistochemistry to analyze $\alpha$-SMA expression in the neuromas.

\section{Toluidine Blue Staining and TEM}

The neuroma specimens were immediately fixed in $2.5 \%$ glutaraldehyde for 2.5 hours, followed by postfixation in $1 \%$ osmium tetroxide for 2 hours, after which the samples were dehydrated in a graded series of ethanol, embedded in epoxy resin, and cut into 1- $\mu \mathrm{m}$ semithin and 50-nm ultrathin sections. The semithin sections were stained with toluidine blue, and the ultrathin sections were used for TEM. Images were acquired from 5 random fields for each sample. All measurements were performed by an assistant who was unaware of the treatment information of each section.

\section{Western Blot Analysis}

The specimens were lysed with lysis buffer $(100 \mathrm{mmol} / \mathrm{L}$ dithiothreitol, $50 \mathrm{mmol} / \mathrm{L}$ Tris- $\mathrm{HCl}, \mathrm{pH} 6.8,2 \%$ sodium dodecyl sulfate, and $10 \%$ glycerol) containing protease inhibitors (Roche). The bicinchoninic acid assay was used to determine total protein concentration. Equal amounts of protein were resolved on a sodium dodecyl sulfate-polyacrylamide gel and then transferred to polyvinylidene difluoride membranes. The membranes were blocked with $5 \%$ nonfat dry milk in TBST buffer for 1 hour. The membranes were washed 3 times with TBST buffer and then incubated with monoclonal antibodies against rabbit antisubstance P (dilution: 1:400, GeneTex, Inc.) and mouse anti-c-fos (dilution: 1:200, Santa Cruz Biotechnology) at room temperature for 1.5 hours. After an additional 3 washes, the samples were incubated for 2 hours at room temperature with horseradish peroxidase-conjugated goat anti-mouse $\operatorname{IgG}(1: 1000)$ or goat anti-rabbit IgG (1:1000). The membranes were then washed with TBST buffer, and the images were scanned with a GS800 Densitometer Scanner. The optical density data were analyzed using Image-Pro Plus software (Media Cybernetics). We used beta-actin (dilution: 1:1000, Santa Cruz Biotechnology) as a control.

\section{ELISA for Serum IL-17 and IL-1 $\beta$}

The serum interleukin (IL) -17 and $-1 \beta$ levels were measured using an ELISA kit (Rat IL-17A Platinum ELISA, Cloud-Clone Corp, Inc.; Rat IL-1 $\beta$ Platinum ELISA, Cusabio, Inc.) according to the manufacturer's protocols. All samples were measured in triplicate, and the mean concentration was calculated from the standard curve. 
TABLE 1. Description of primers used in RT-PCR

\begin{tabular}{lllc}
\hline \multicolumn{1}{c}{ Gene } & \multicolumn{1}{c}{ Forward Primer } & \multicolumn{1}{c}{ Reverse Primer } & Size (bp) \\
\hline RhoA & 5'-CTGGAGAAACCTGCCAAGTATG-3' $^{\prime}$ & 5'-GGTGGAAGAATGGGAGTTGCT-3' $^{\prime}$ & 138 \\
\hline GAPDH & 5'AGCACACAAGGCGGGAGTTA-3' $^{\prime}$ & 5'-CTCCGTCTTTGGTCTTTGCTGA-3' $^{\prime}$ & 126 \\
\hline
\end{tabular}

\section{Quantitative RT-PCR}

Total RNA was extracted from the stored specimens using an RNeasy Mini Kit (QIAGEN) according to the manufacturer's protocol. The amount of RNA was determined using a NanoDrop UV Spectrophotometer (NanoDrop Technologies). cDNA was synthesized using a RevertAid First Strand cDNA Synthesis Kit (Thermo Fisher Scientific), and RT-PCR was performed using FastStart Universal SYBR Green Master (Rox) (Roche Life Science) on an Applied Biosystems StepOne RT-PCR system. All reactions were run in triplicate. The relative expression of each mRNA was calculated with the comparative $\Delta \Delta \mathrm{CT}$ method and was normalized against the housekeeping gene GAPDH. The primer information is listed in Table 1.

\section{Statistical Analysis}

The mean \pm SD was used to describe the data, and the data were analyzed using SPSS version 20.0 (IBM Corp.). Differences among groups were evaluated by one-way analysis of variance, and the Bonferroni method was used to compare differences between two different groups. Any $\mathrm{p}<0.05$ was considered statistically significant.

\section{Results}

\section{Evaluation of Neuroma Appearance and Weight}

Two weeks postoperatively, slightly bulb-like neuromas could be found at the end of the PNS in the positive group, while no typical neuromas were observed in the blank group or CSPG group; 8 weeks postoperatively, typical bulbous neuromas were observed in the positive group. In contrast, no rats in the blank group or the CSPG group developed bulbous neuromas after neurotomy (Fig. 3).

\section{Autotomy Behavior Evaluation}

Autotomy behavior was only observed on the right foot (operative side). Statistically significant differences in the average autotomy scores were found, and autotomy behavior was obviously better in the CSPG group than in the positive group and the blank group $(\mathrm{p}<0.05$ and $\mathrm{p}<0.01$, respectively), except at the end of the first 3 weeks, when the autonomy behavior was similar in the blank and CSPG groups ( $>$ > 0.05) (Fig. 3).

\section{Masson's Trichrome Staining and Immunohistochemistry for $\alpha$-SMA}

Masson's trichrome staining showed blue-stained collagenous fibers in the neuromas (Fig. 4). In the positive group, the structure of most neuromas was extremely chaotic. A large number of blue-stained collagenous fibers were irregular and disorganized, and most of the collagenous fibers were agglomerated. In addition, we observed that muscles grew into the neuroma. In the blank group and CSPG group, the distribution of collagenous fibers was relatively regular; most of the collagenous fibers were scattered and thin, and only a few collagenous fibers were gathered together.

$\alpha$-SMA expression in the neuromas was analyzed by immunohistochemistry (Fig. 4). Brown staining indicates $\alpha$-SMA expression. We observed markedly increased $\alpha$ SMA expression in the positive group compared with that in the blank and CSPG groups.

\section{Silver Staining}

Silver staining showed that the regenerated axons (stained in black) were densely distributed in a chaotic manner in the positive and blank groups, including many transverse and longitudinal regenerated axons, while there were only a small number of transverse regenerated axons in the CSPG group compared with the other two groups, indicating that the regenerated axons were more regular in the CSPG group (Fig. 5).

\section{Toluidine Blue Staining and TEM}

Eight weeks postoperatively, among the groups, the CSPG group showed the best myelination of the regenerated axons (Fig. 5). We measured the diameter of the myelinated axons and the thickness of the new myelin sheath in the TEM images and found that the diameter of the myelinated axons and the thickness of the myelin were significantly increased in the CSPG group compared with those in the other two groups $(\mathrm{p}<0.01)$, and the differences between the positive group and the blank group were not significant $(\mathrm{p}>0.05)$.

\section{Expression of Pain-Related Markers}

The expression levels of substance $\mathrm{P}, \mathrm{c}$-fos, and serum IL-17 and IL-1 $\beta$ are considered to be pain-related markers after peripheral nerve neurotomy (Fig. 6). Two weeks postoperatively, the expression levels of substance P, c-fos, and serum IL-17 and IL- $1 \beta$ were significantly decreased in the CSPG group compared with those in the positive group and the blank group $(\mathrm{p}<0.01$ and $\mathrm{p}<0.05$, respectively). Additionally, 8 weeks postoperatively, the expression levels of substance P and IL-17 were still significantly lower in the CSPG group than those in the positive and blank groups $(\mathrm{p}<0.01$ and $\mathrm{p}<0.05)$, while the $\mathrm{c}$-fos and IL-1 $\beta$ levels showed no significant differences among the three groups.

\section{RhoA Gene Expression in Bilateral L4-5 DRG}

Two weeks postoperatively, RhoA gene expression in the L4-5 DRG was dramatically upregulated on the operated side compared with that on the contralateral unin- 



FIG. 3. Observation of neuroma growth and autotomy behavior. A: Typical bulbous neuroma in the positive group 8 weeks postoperatively. B and C: Atypical linear neuromas in the blank and CSPG groups. D: Statistical analysis of the weight ratio in the three groups (\#p < 0.05, \#\#p < 0.01). E: No autotomy behavior of the toes (0 points). F: The nails on the fourth and fifth toes were chewed (1 point). G: The distal digit on the fifth toe and the nails on the fourth and fifth toes were chewed ( 3 points). H: The distal digits on the third, fourth, and fifth toes and the nails on the fourth and fifth toes were chewed (7 points). I: Statistically significant differences in the mean autotomy scores were found; there was obvious improvement in the autotomy behavior in the CSPG group compared with that in the positive and blank groups (\#p <0.05,\#\#p<0.01), except at the end of the first 3 weeks in the blank group, at which time the autonomy behavior was similar to that in the CSPG group (\&p > 0.05). Figure is available in color online only.

jured side in all groups $(\mathrm{p}<0.01)$, and the CSPG group showed the most obvious upregulation. Eight weeks postoperatively, RhoA expression in the L4-5 DRG was significantly upregulated on the operated side compared with that on the contralateral uninjured side in the CSPG group, but there was no such significant difference in the positive or blank group $(\mathrm{p}<0.01)$ (Fig. 6$)$.

\section{Discussion}

Traumatic neuroma caused by nerve injuries or amputation is an important source of neuropathic pain. ${ }^{3,20} \mathrm{Al}-$ though the exact mechanisms by which traumatic neuromas cause pain-related symptoms are still unknown, various methods are used to stop traumatic painful neuroma formation. Generally, surgeons perform direct neuroma excision and then suture the nerve stump to the nearby muscle or vein, and this approach is currently the most commonly used strategy to prevent traumatic neuroma formation in the clinic, which can achieve satisfactory results. ${ }^{12}$ However, when there is no appropriate bone or muscle at the site of the nerve stump, muscle or bone capping may not be useful. ${ }^{13}$ Another method, called "centrocentral anastomosis," is used to prevent traumatic neuroma formation by pairing fascicular groups of the proximal stump of a severed nerve, but its application is limited by the complexity of the operation, and it may not be suitable for very small nerves..$^{21}$

With the development of tissue engineering, there are many emerging materials for the treatment of peripheral nerve injury and for the prevention of traumatic painful neuromas. Studies have shown that silicone tubes, collagen tubes, microcrystalline chitosan, and PRGD/PDLLA conduits can achieve satisfactory effects in preventing traumatic neuroma formation..$^{22-28}$ Hong et al. ${ }^{29}$ found that acellular nerve allografts (ANAs) attached to the proximal end of an injured nerve can limit axon growth in a controlled manner, indicating that the use of ANAs as a tool to control neuroma formation may be a viable clinical option. In addition, Zhou et al. ${ }^{11}$ found that the application of a nanofibrous scaffold consisting of aligned silk fibroin blended with poly(L-lactic acid-co-caprolactone) activated the $R h o A /$ ROCK signaling pathway, which contributed to the prevention of traumatic painful neuroma formation because of its suppressive effect on axon regeneration. Although the exact mechanisms by which these capping techniques prevent traumatic painful neuroma formation need to be further investigated, we can preliminarily conclude that the lower density and limited distribution of dis- 

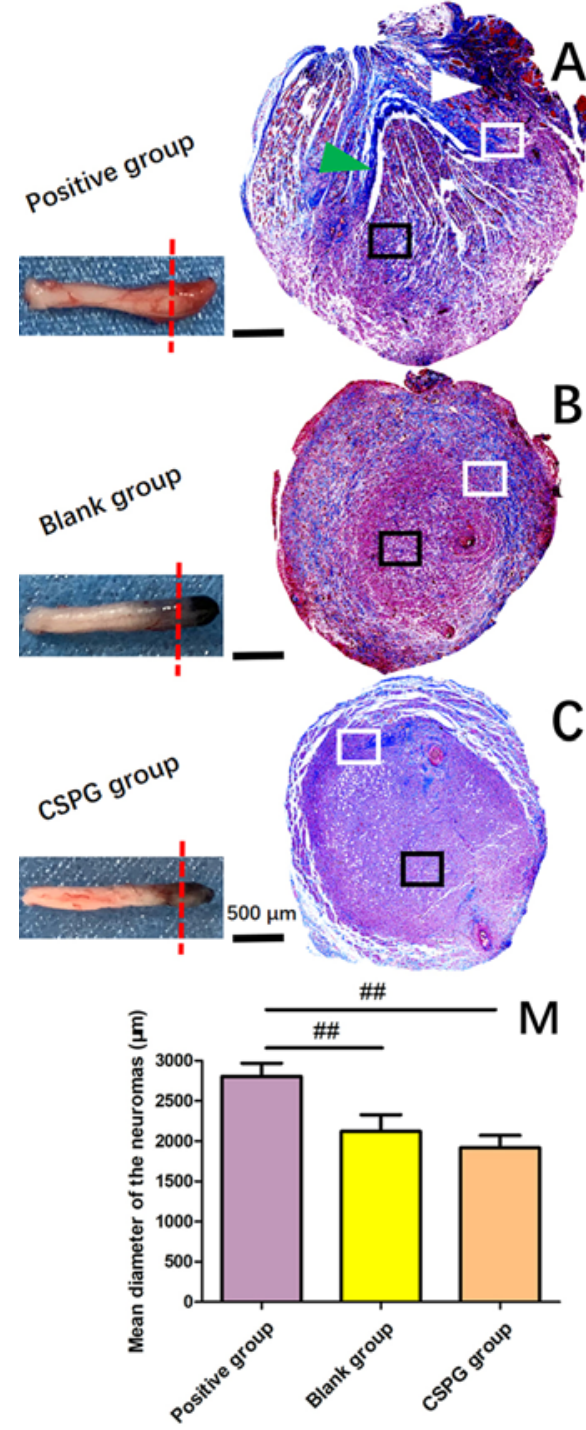
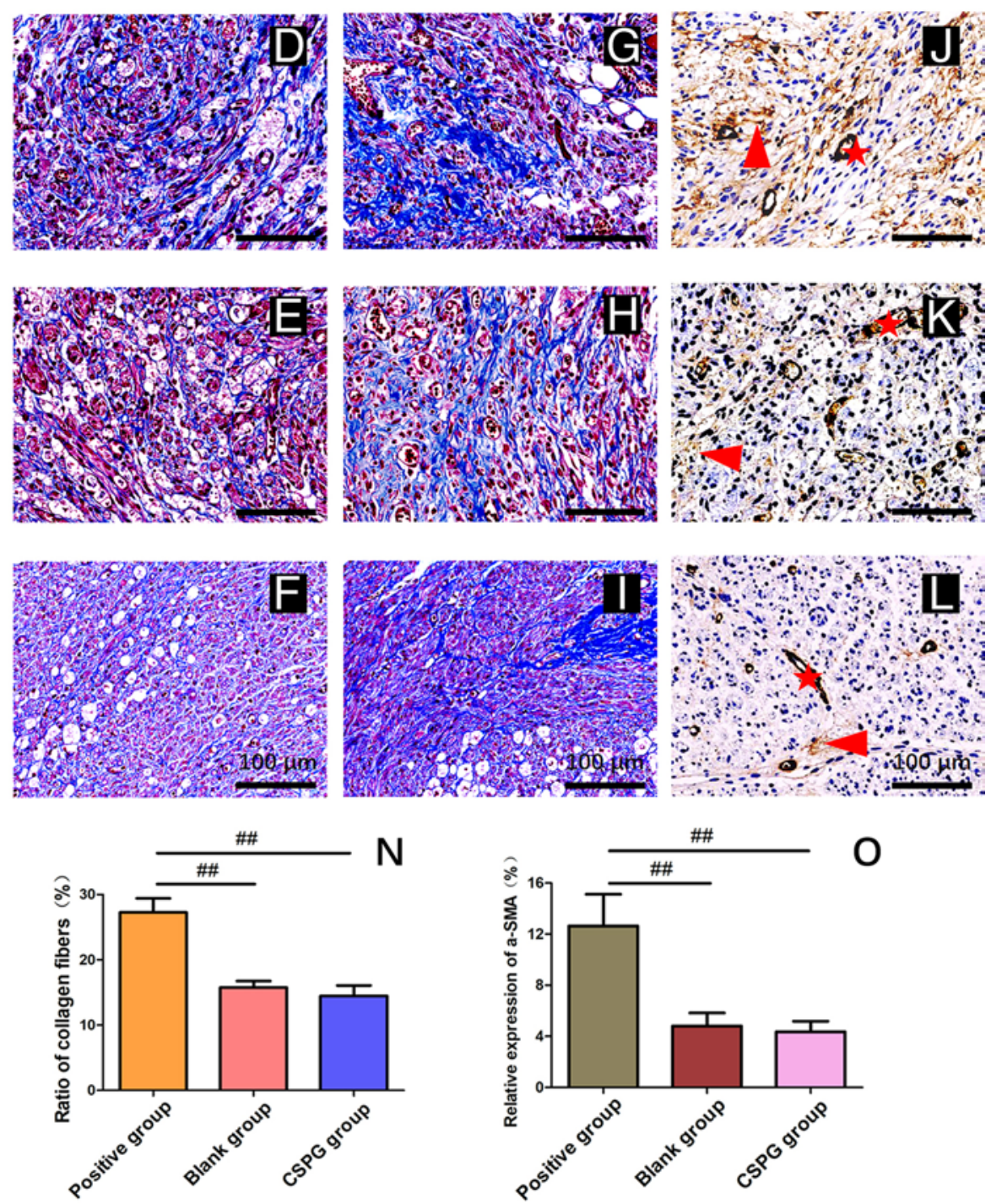

FIG. 4. The results of Masson's trichrome staining of collagenous fibers and immunohistochemistry for $\alpha$-SMA expression in neuromas. D-F were captured from the black boxes in A-C around the central area, respectively. $\mathrm{G}-\mathrm{I}$ were captured from the white boxes in $\mathrm{A}-\mathrm{C}$ around the marginal area, respectively. A, D, and G: A typical neuroma in the positive group, in which there were many blue-stained collagenous fibers that were irregular and disorganized, and most of the collagenous fibers were agglomerated (green arrowhead). The white arrowhead shows muscles near the neuroma inserting into the neuroma. In the blank (B, E, and $\mathrm{H})$ and CSPG (C, F, and I) groups, the distribution of the collagenous fibers was relatively regular, and most of the fibers were scattered and thin. In both blocker groups, only a few collagenous fibers were gathered together. In addition, in both the central and peripheral areas, the distribution and density of collagenous fibers were more extensive in the positive group than in the blank and CSPG groups. J-L: Results of $\alpha$-SMA immunohistochemistry. Brown staining indicates $\alpha$-SMA expression (red arrowheads), and puce staining indicates strong $\alpha$-SMA expression in vascular smooth muscle (red stars). J: A large amount of agglomerated $\alpha$-SMA was dispersed in the positive group. $\mathrm{K}$ and $\mathrm{L}$ : In the blank and CSPG groups, there was very little $\alpha$-SMA expression. M-0: Statistical analysis of the mean diameter of the neuromas, the ratio of collagenous fibers, and the relative expression of $\alpha$-SMA in the three groups, respectively $(\# \# p<0.01)$. Figure is available in color online only.

orderly collagenous fibers and irregular regenerated axons at the injury site are beneficial to prevent traumatic painful neuroma formation.

Therefore, we endeavored to investigate more effective and specific methods to block irregular axon regeneration and inhibit disorderly collagenous fiber accumulation to prevent traumatic painful neuroma formation. Our previous studies found that spatial distribution affects the role of CSPGs in axon regeneration; when the growth cone extending from the DRG was covered with CSPGs, neurite outgrowth was inhibited, and neurite density was decreased, while these effects were not observed when the soma or the proximal axons were covered with CSPGs. ${ }^{30}$ Thus, we developed a new strategy to prevent the formation of traumatic painful neuromas by applying a CSPGgelatin blocker to the nerve stump after neurotomy. We also found that CSPGs loaded in the gelatin blocker can proactively and directly block and inhibit axon outgrowth 


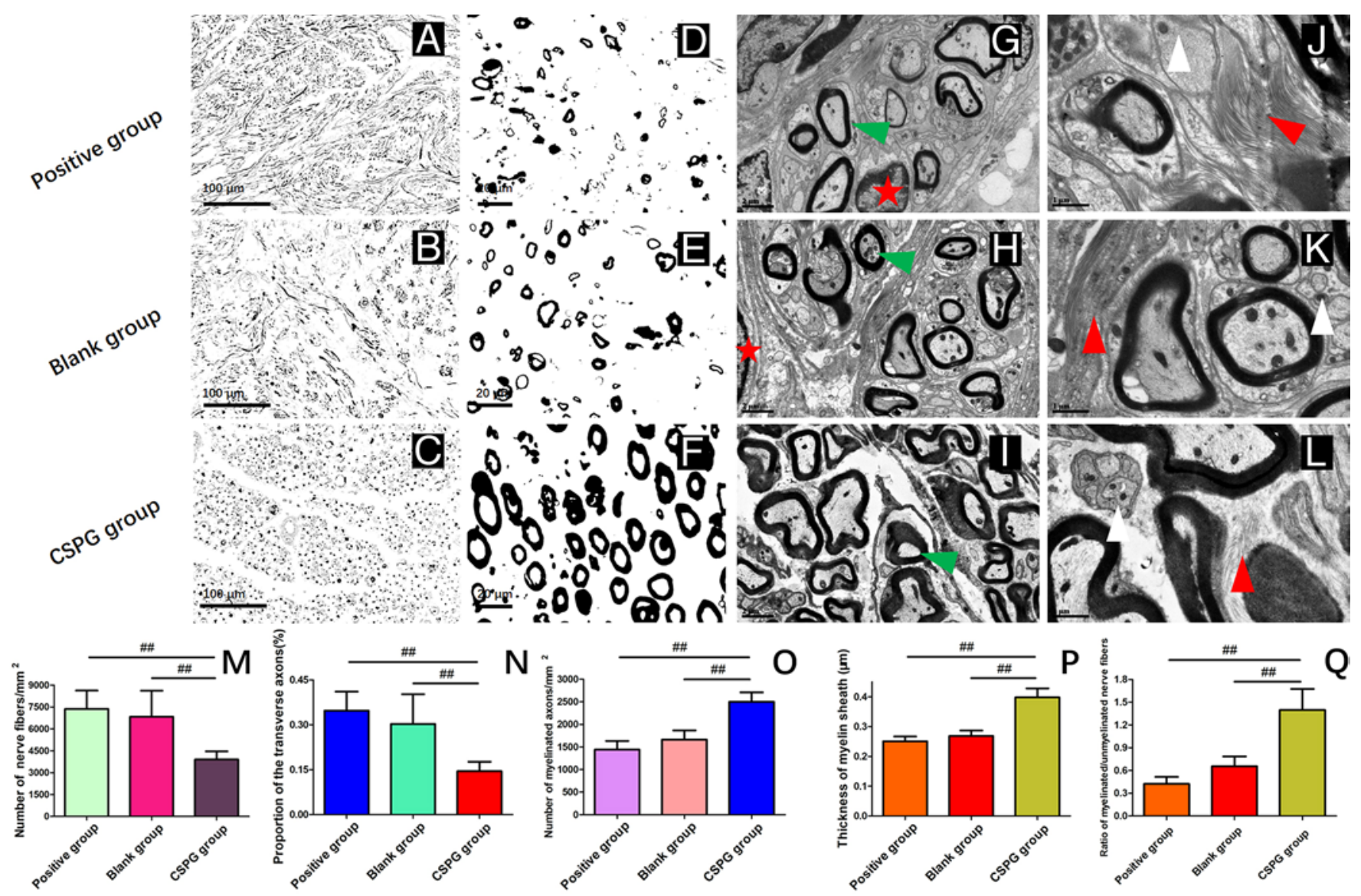

FIG. 5. A-C: The results of silver staining of nerve fibers in the neuromas (stained in black). In the positive (A) and blank (B) groups, many transverse and longitudinal nerve fibers were randomly and irregularly distributed in the neuroma. In the CSPG group (C), in which most of the nerve fibers were longitudinal, only a few nerve fibers were transverse. In addition, in the positive and blank groups, the nerve fibers tended to be congregated, while there was no such tendency in the CSPG group. D-F: Toluidine blue staining shows that obviously fewer and thinner blue-stained myelin sheaths were present in the positive group (D) and the blank group (E) than in the CSPG group (F). G-L: TEM images of samples from the three groups. The green arrowheads show thick myelin sheaths, the white arrowheads show unmyelinated myelin sheaths, the red arrowheads show collagenous fibers in the neuromas, and the red stars show fibroblasts in the positive and blank groups. We observed more thick myelin sheaths and fewer thin or unmyelinated myelin sheaths in the CSPG group than in the positive and blank groups. In addition, the distribution of collagenous fibers was more disordered and the fibers were denser in the positive group than in the blank and CSPG groups. M-Q: Statistical analysis of the number of nerve fibers, the proportion of transverse nerve fibers, the number of myelinated axons, the thickness of myelin sheath, and the ratio of myelinated/unmyelinated axons in the three groups, respectively (\#\# < 0.01).

Figure is available in color online only.

and elongation. Once the regenerating axons grew into the gelatin microchannels, the CSPGs loaded in the pore wall could completely cover the growth cone of the regenerated axons and block their outgrowth and elongation; thus, axon regeneration could be confined to the gelatin microchannels, and the axons could not extend and regenerate in all directions. In addition, Ughrin et al. ${ }^{31}$ found that CSPGs can lead to growth cone collapse, further decreasing the density of regenerated axons. Our results confirm that axon regeneration was lower in density and more regular in arrangement in the CSPG group than in the positive and blank groups. RhoA gene expression also confirmed the inhibitory effects of CSPGs. At 2 and 8 weeks postoperatively, the most significant upregulation of RhoA gene expression was observed in the CSPG group, which could be due to binding of the CSPGs to receptors in the growth cone, such as NgR1 and NgR3. In addition, more regu- lar and less dense regenerated axons may be beneficial for improved myelination, as we observed better and thicker myelination on the regenerated axons in the CSPG group than on those in the positive and blank groups, which is also beneficial for preventing traumatic painful neuroma formation because neuropathic pain caused by traumatic neuromas has been reported to be primarily mediated by unmyelinated fibers. ${ }^{8,32}$

Another important aspect of this study is that we applied gelatin as the carrier material to be loaded with CSPGs. As a biodegradable material with good biocompatibility, gelatin has been widely used in tissue engineering, drug delivery, and in vitro 3D cell culture. Studies have shown that gelatin-based hydrogels can effectively be loaded with and control the release of vascular endothelial growth factor. ${ }^{33}$ Additionally, gelatin-based conduits can prevent the infiltration of fibroblasts and reduce the risk of scar tissue 


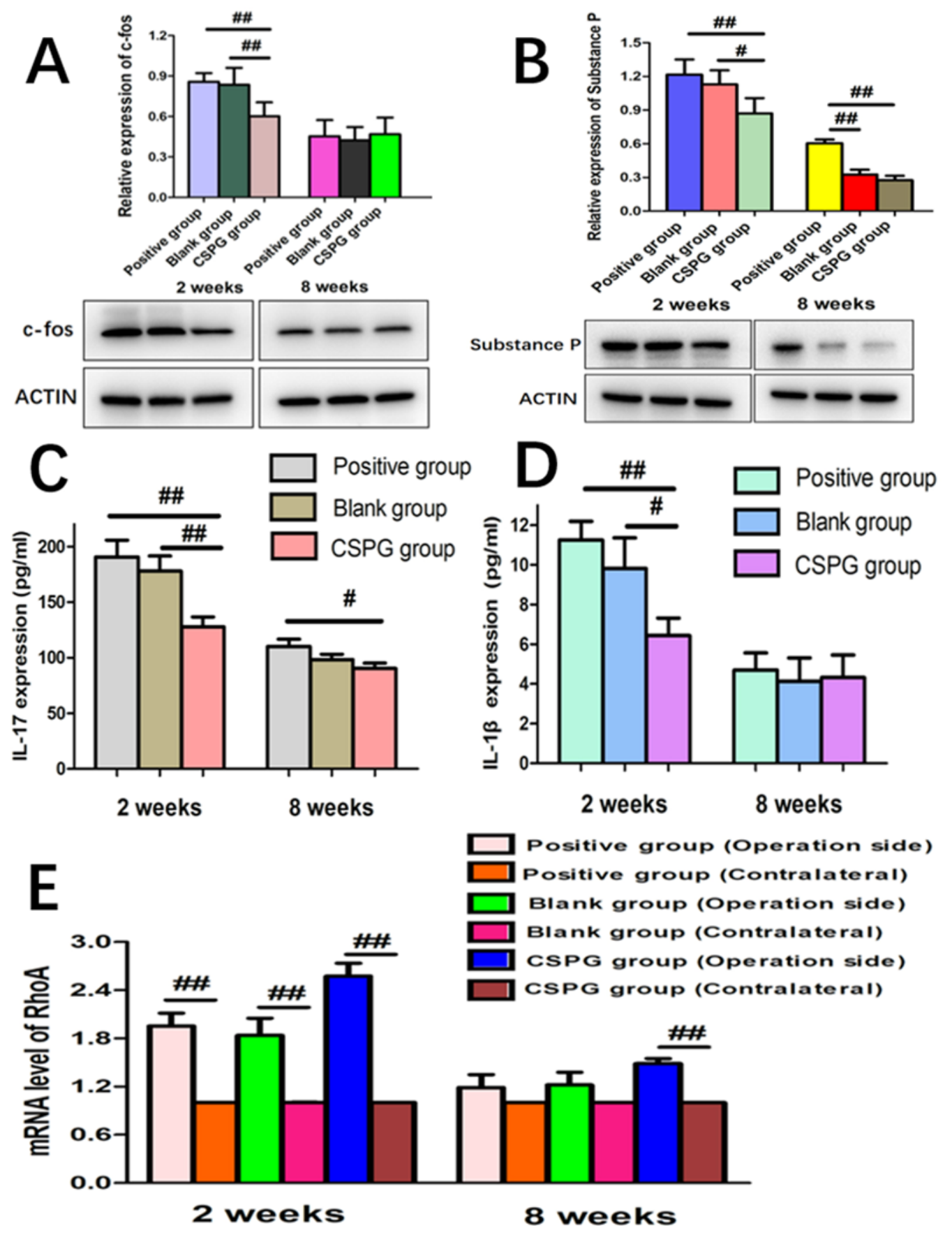

FIG. 6. Results of pain-related marker and RhoA gene expression. A and B: Western blot analysis of c-fos and substance P. C and D: ELISA test for serum IL-17 and IL-1 $\beta$. E: RhoA gene expression in bilateral L4-5 DRG. \#p < 0.05, \#\#p $<0.01$. Figure is available in color online only.

formation, which could provide an advantageous environment for nerve regeneration. ${ }^{34}$ More importantly, gelatin forms a polyporous structure after lyophilization, which can obviously increase the contact area between CSPGs and growth cones, contributing to the improved inhibitory effect of CSPGs. Therefore, we found that the distribution of the collagenous fibers was more structured and more linear and that the fibers were less dense in the blank and CSPG groups than in the positive group, and similar patterns were observed for the expression of $\alpha$-SMA. These findings indicate that the gelatin blocker could protect the nerve stump and decrease the accumulation of collagenous fiber at the injury site after neurectomy. However, the simple gelatin shell of the blocker could not stop the growth of regenerating axons. Axons could penetrate the gelatin shell and grow randomly in all directions, which may be the reason why the regenerating axons in the blank group were irregular and disorganized and why the simple gelatin blocker could not achieve satisfactory effects to prevent the formation of traumatic painful neuromas. 
Overall, the combined application of gelatin blockers and CSPGs can not only inhibit the disorderly accumulation of collagenous fibers but also block the irregular regeneration of axons, thus fundamentally preventing traumatic neuroma formation.

There are some additional issues that need to be discussed. After sciatic nerve neurotomy, terminal neuromas may form, and afferent fibers in such neuromas spontaneously emit ectopic input that coincides with the outbreak of licking, scratching, and self-mutilation of the denervated limb, leading to autotomy behavior. ${ }^{19}$ Autotomy behavior is considered to be the expression of spontaneously disagreeable sensations, such as paresthesia, dysesthesia, and neuropathic pain; thus, autotomy behavior has been adopted as a parameter for evaluating pain in rats. ${ }^{11,25}$ However, this behavior is an indirect assessment of pain, and more specific pain-related symptoms are required for further study. On the other hand, the expression of c-fos in the lumbar spinal cord and substance $\mathrm{P}$ in the neuromas was significantly decreased when terminal neuroma formation was suppressed in other previous studies. ${ }^{35,36}$ Regarding the serum IL-17 and IL-1 $\beta$ levels, studies have found that they are related to neuropathic pain; thus, they are usually considered indicators of the pain status during a continuous phase after neurotomy. ${ }^{37,38}$ To better reflect the pain status in rats after neurotomy in our study, we chose c-fos, substance $P$, and serum IL-17 and IL-1 $\beta$ as pain-related markers after neurotomy. Our results show that c-fos, substance $\mathrm{P}$, and serum IL-17 and IL-1 $\beta$ were all significantly downregulated in the CSPG group 2 weeks postoperatively, which is in line with the results of previous studies. Regarding substance P and IL-17, there were significant differences between the positive and CSPG groups at 8 weeks postoperatively. Neuroma-related pain is a chronic problem, and substance $\mathrm{P}$ and IL-17 play important roles in chronic neuropathic pain, ${ }^{36}$ which can explain the results at 8 weeks postoperatively. Therefore, our results clearly show that the application of CSPG-gelatin blockers to the PNS after neurotomy can effectively improve autotomy behavior and relieve pain.

The present study has some limitations. The first is the short-term follow-up period. Even though evaluation periods of 8 weeks or less have been set as the endpoint in different studies on traumatic neuromas, ${ }^{11,25,29,39,40}$ a longer evaluation period for painful neuromas may be better. This is not only because neuroma-related pain is a chronic problem but also because it is necessary to evaluate the long-term effects after the gelatin blocker completely degrades. Another limitation is the concentration of CSPGs. Studies have shown that the inhibition of neurite outgrowth by CSPGs is concentration dependent. Snow and Letourneau ${ }^{41}$ found that CSPGs at a concentration of 40 $\mu \mathrm{g} / \mathrm{ml}$ resulted in approximately 50\% elongation onto CSPGs and $50 \%$ inhibition, in contrast to $1000 \mu \mathrm{g} / \mathrm{ml}$, which resulted in complete inhibition in vitro. Moreover, Zou et al. ${ }^{30}$ found that, compared with the control condition, CSPGs $(10 \mu \mathrm{g} / \mathrm{ml})$ directly covering the growth cone significantly inhibited neurite elongation and reduced the neurite density. A high concentration of CSPGs may lead to an inflammatory response in the body ${ }^{42}$ Therefore, in our study, we chose $20-\mu \mathrm{g} / \mathrm{ml} \mathrm{CSPGs} \mathrm{dissolved} \mathrm{in} \mathrm{a} \mathrm{gela-}$ tin solution. In further study, additional subgroups with different CSPG concentrations will be established to study the dose-effect relationship of exogenous CSPGs in vivo.

\section{Conclusions}

This study provides new insight and a new strategy to prevent traumatic painful neuroma formation by artificially regulating the spatial distribution of CSPGs in gelatin, which not only reduces the disorderly accumulation of scar tissue and protects the PNS but also proactively and effectively blocks irregular axon regeneration and improves axon myelination, followed by the obvious relief of the pain symptoms caused by traumatic neuromas. Overall, covering the PNS with CSPGs may be a potential and promising method to prevent traumatic painful neuroma formation in the clinic.

\section{Acknowledgments}

This study was supported by grants from the National Key Research and Development Plan of China (no. 2016YFC1101603 to X.L.L.), NSFC (no. 31670986 to Q.T.Z.), the Science and Technology Project of Guangdong Province of China (nos. 2014B020227001 and 2017A050501017 to Q.T.Z.), and the Science and Technology Project of Guangzhou of China (no. 201807010082 to Q.T.Z.).

\section{References}

1. Cahn LR. Traumatic (amputation) neuroma. Am J Orthod Oral Surg. 1939;25:190-193.

2. Finnerup NB, Haroutounian S, Kamerman P, et al. Neuropathic pain: an updated grading system for research and clinical practice. Pain. 2016;157(8):1599-1606.

3. Foltán R, Klíma K, Spacková J, Sedý J. Mechanism of traumatic neuroma development. Med Hypotheses. 2008;71(4):572-576.

4. Domeshek LF, Krauss EM, Snyder-Warwick AK, et al. Surgical treatment of neuromas improves patient-reported pain, depression, and quality of life. Plast Reconstr Surg. 2017;139(2):407-418.

5. Fayad JN, Linthicum FH Jr. Symptomatic postsurgical traumatic neuromas. Otol Neurotol. 2009;30(7):981-984.

6. van der Avoort DJ, Hovius SE, Selles RW, et al. The incidence of symptomatic neuroma in amputation and neurorrhaphy patients. J Plast Reconstr Aesthet Surg. 2013;66(10):1330-1334.

7. Khan N, Smith MT. Neurotrophins and neuropathic pain: role in pathobiology. Molecules. 2015;20(6):10657-10688.

8. Mackinnon SE, Dellon AL, Hudson AR, Hunter DA. Alteration of neuroma formation by manipulation of its microenvironment. Plast Reconstr Surg. 1985;76(3):345-353.

9. Marcol W, Kotulska K, Larysz-Brysz M, Kowalik JL. BDNF contributes to animal model neuropathic pain after peripheral nerve transection. Neurosurg Rev. 2007;30(3):235-243.

10. Yan H, Gao W, Pan Z, et al. The expression of $\alpha$-SMA in the painful traumatic neuroma: potential role in the pathobiology of neuropathic pain. J Neurotrauma. 2012;29(18):2791-2797.

11. Zhou X, Zhao B, Poonit K, et al. An aligned nanofiber nerve conduit that inhibits painful traumatic neuroma formation through regulation of the RhoA/ROCK signaling pathway. $J$ Neurosurg. 2019;132(3):837-846.

12. Poppler LH, Parikh RP, Bichanich MJ, et al. Surgical interventions for the treatment of painful neuroma: a comparative meta-analysis. Pain. 2018;159(2):214-223.

13. Yao C, Zhou X, Zhao B, et al. Treatments of traumatic 
neuropathic pain: a systematic review. Oncotarget. 2017;8(34):57670-57679.

14. Erskine L, Herrera E. The retinal ganglion cell axon's journey: insights into molecular mechanisms of axon guidance. Dev Biol. 2007;308(1):1-14.

15. Treloar HB, Nurcombe V, Key B. Expression of extracellular matrix molecules in the embryonic rat olfactory pathway. $J$ Neurobiol. 1996;31(1):41-55.

16. Dickendesher TL, Baldwin KT, Mironova YA, et al. NgR1 and NgR3 are receptors for chondroitin sulfate proteoglycans. Nat Neurosci. 2012;15(5):703-712.

17. Walker BA, Ji SJ, Jaffrey SR. Intra-axonal translation of RhoA promotes axon growth inhibition by CSPG. J Neurosci. 2012;32(41):14442-14447.

18. Wall PD, Scadding JW, Tomkiewicz MM. The production and prevention of experimental anesthesia dolorosa. Pain. 1979;6(2):175-182.

19. Zeltser R, Beilin B, Zaslansky R, Seltzer Z. Comparison of autotomy behavior induced in rats by various clinically-used neurectomy methods. Pain. 2000;89(1):19-24.

20. Buch NS, Qerama E, Finnerup NB, Nikolajsen L. Neuromas and postamputation pain. Pain. 2020;161(1):147-155.

21. Barberá J, Albert-Pampló R. Centrocentral anastomosis of the proximal nerve stump in the treatment of painful amputation neuromas of major nerves. J Neurosurg. 1993;79(3):331334.

22. Economides JM, DeFazio MV, Attinger CE, Barbour JR. Prevention of painful neuroma and phantom limb pain after transfemoral amputations through concomitant nerve coaptation and collagen nerve wrapping. Neurosurgery. 2016;79(3):508-513.

23. Onode E, Uemura T, Takamatsu K, et al. Nerve capping with a nerve conduit for the treatment of painful neuroma in the rat sciatic nerve. J Neurosurg. 2019;8:1-9.

24. Fathi HR, Fathi M, Ghannadan A, et al. The healing effect of silicone gel on sciatic nerve injuries in experimental rat. World J Plast Surg. 2014;3(2):93-98.

25. Yi J, Jiang N, Li B, et al. Painful terminal neuroma prevention by capping PRGD/PDLLA conduit in rat sciatic nerves. Adv Sci (Weinh). 2018;5(6):1700876.

26. Kim PD, Hayes A, Amin F, et al. Collagen nerve protector in rat sciatic nerve repair: a morphometric and histological analysis. Microsurgery. 2010;30(5):392-396.

27. Marcol W, Larysz-Brysz M, Kucharska M, et al. Reduction of post-traumatic neuroma and epineural scar formation in rat sciatic nerve by application of microcrystallic chitosan. Microsurgery. 2011;31(8):642-649.

28. Sakai Y, Ochi M, Uchio Y, et al. Prevention and treatment of amputation neuroma by an atelocollagen tube in rat sciatic nerves. J Biomed Mater Res B Appl Biomater. 2005;73(2):355-360.

29. Hong T, Wood I, Hunter DA, et al. Neuroma management: capping nerve injuries with an acellular nerve allograft can limit axon regeneration. Hand $(N Y)$. Published online May 29, 2019. doi:10.1177/1558944719849115

30. Zou JL, Sun JH, Qiu S, et al. Spatial distribution affects the role of CSPGs in nerve regeneration via the actin filamentmediated pathway. Exp Neurol. 2018;307:37-44.

31. Ughrin YM, Chen ZJ, Levine JM. Multiple regions of the NG2 proteoglycan inhibit neurite growth and induce growth cone collapse. J Neurosci. 2003;23(1):175-186.
32. Ueda H. Peripheral mechanisms of neuropathic pain-involvement of lysophosphatidic acid receptor-mediated demyelination. Mol Pain. 2008;4:11.

33. Gnavi S, di Blasio L, Tonda-Turo C, et al. Gelatin-based hydrogel for vascular endothelial growth factor release in peripheral nerve tissue engineering.J Tissue Eng Regen Med. 2017;11(2):459-470.

34. Tao J, Hu Y, Wang S, et al. A 3D-engineered porous conduit for peripheral nerve repair. Sci Rep. 2017;7:46038.

35. Ahmad AH, Ismail Z. c-fos and its consequences in pain. Malays J Med Sci. 2002;9(1):3-8.

36. Zieglgänsberger W. Substance $\mathrm{P}$ and pain chronicity. Cell Tissue Res. 2019;375(1):227-241.

37. Gui WS, Wei X, Mai CL, et al. Interleukin-1 $\beta$ overproduction is a common cause for neuropathic pain, memory deficit, and depression following peripheral nerve injury in rodents. Mol Pain. Published online May 12, 2016. doi:10.1177/1744806916646784

38. Sun C, Zhang J, Chen L, et al. IL-17 contributed to the neuropathic pain following peripheral nerve injury by promoting astrocyte proliferation and secretion of proinflammatory cytokines. Mol Med Rep. 2017;15(1):89-96.

39. Bolleboom A, de Ruiter GCW, Coert JH, et al. Novel experimental surgical strategy to prevent traumatic neuroma formation by combining a 3D-printed Y-tube with an autograft. $J$ Neurosurg. 2018;130(1):184-196.

40. Yan H, Zhang F, Kolkin J, et al. Mechanisms of nerve capping technique in prevention of painful neuroma formation. PLoS One. 2014;9(4):e93973.

41. Snow DM, Letourneau PC. Neurite outgrowth on a step gradient of chondroitin sulfate proteoglycan (CS-PG). J Neurobiol. 1992;23(3):322-336.

42. Rolls A, Shechter R, London A, et al. Two faces of chondroitin sulfate proteoglycan in spinal cord repair: a role in microglia/macrophage activation. PLoS Med. 2008;5(8):e171.

\section{Disclosures}

The authors report no conflict of interest concerning the materials or methods used in this study or the findings specified in this paper.

\section{Author Contributions}

Conception and design: Zhu, He, Qiu, Zou. Acquisition of data: $\mathrm{He}, \mathrm{Gu}, \mathrm{Yao}, \mathrm{Tu}$. Analysis and interpretation of data: He, Qiu, Gu, Yao, Tu. Drafting the article: He, Tu, Wang. Critically revising the article: Zhu, Qiu, Zou, Liu, Zhou. Reviewed submitted version of manuscript: Zhu, Qiu, Zou, Wang, Liu, Zhou. Approved the final version of the manuscript on behalf of all authors: Zhu. Statistical analysis: Qiu, Zou. Administrative/technical/material support: Zhu, Zou, Gu, Yao, Tu, Wang, Liu, Zhou. Study supervision: Zhu, Liu, Zhou.

\section{Correspondence}

Qing-Tang Zhu: The First Affiliated Hospital of Sun Yat-sen University, Guangzhou, People's Republic of China. zhuqingt@ mail.sysu.edu.cn. 